Ibrahim, M., Berghede, K., Thomack, D., Lampsas, P., Kievet D., and Hanna, A.. (2017). “A Framework of FiveStream Production System for Megaprojects” In: LC3 2017 Volume II - Proceedings of the 25th Annual Conference of the International Group for Lean Construction (IGLC), Walsh, K., Sacks, R., Brilakis, I. (eds.), Heraklion, Greece, pp. 729-736. DOI: https://doi.org/10.24928/2017/0179

\title{
A FRAMEWORK OF FIVE-STREAM PRODUCTION SYSTEM FOR MEGAPROJECTS
}

\author{
Michael W. Ibrahim ${ }^{1}$, Klas Berghede ${ }^{2}$, David Thomack ${ }^{3}$, Panos Lampsas ${ }^{4}$, Dave Kievet ${ }^{5}$, \\ and Awad S. Hanna ${ }^{6}$
}

\begin{abstract}
Construction projects, particularly megaprojects, tend to frequently fail in satisfying their budget, schedule, quality, and safety objectives. Two of the most notable systems that have been developed to enhance project performance are the Last Planner ${ }^{\circledR}$ System and Location-Based Management System. This paper builds on combining these two systems through proposing an integrative production system that can play a significant role in enhancing project performance, especially in the case of megaprojects. The proposed production system incorporates five cohesive streams: production planning, material flow, Built in Quality (BIQ)/Information flow, tracking flow, and safety flow. This paper discusses each of these five streams, at five different implementation levels throughout the project lifecycle, in the context of an ongoing successful megaproject. Finally, the paper concludes by listing four main success pillars that are needed for the proposed production system to flourish.
\end{abstract}

Keywords: Lean construction; Production system; Megaprojects; Case study; Integrated Lean Project Delivery ${ }^{\circledR}$ (ILPD)

\section{INTRODUCTION}

While the construction industry makes up a significant portion of the U.S. economy, it is fraught with waste and inefficiencies. Despite mounting technological developments, construction productivity has decreased by nearly 30\% between 1964 and 2011, while nonfarm industries have more than doubled their productivity over the same period (Hanna et al. 2016; Ibrahim 2016). Because of the declining productivity, around $45 \%$ of construction projects are not delivered on time (Hanna 2016). Such losses are even more visible in megaprojects. Recent studies have demonstrated that for megaprojects: 1) $98 \%$ experience cost overruns or delays, 2) the average cost increase is $80 \%$, and 3) the average schedule slippage is 20 months (Changali et al. 2015). Such losses and failures have urged the development of new construction production systems that aim at eliminating the incorporated waste and maximizing the value of the construction process, most notably

1 Doctoral Student, Dept. of Civil and Environmental Engineering, Univ. of Wisconsin-Madison, 2256 Engineering Hall, 1415 Engineering Dr., Madison, WI 53706. E-mail: mwwilliam@wisc.edu

2 Senior Production Manager, The Boldt Company, Western Operations, 2150 River Plaza Drive, Suite 255, Sacramento, CA 95833. E-mail: klas.bergheded@Boldt.com

3 Vice President and General Manager, The Boldt Company, 455 Market Street, Suite 1670, San Francisco, CA 94105. E-mail: david.thomack@Boldt.com

4 Regional Program Manager, Sutter Health, 1200 Van Ness Ave, San Francisco, CA 94109. E-mail: lampsasp@sutterhealth.org

5 Division President for Western and Southern Division at The Boldt Company, 2525 N. Roemer Road, Appleton, WI 54911. E-mail: dave.kievet@boldt.com

6 Professor and Chair, Construction Engineering and Management, Univ. of Wisconsin-Madison, 2320 Engineering Hall, 1415 Engineering Dr., Madison, WI 53706. E-mail: ashanna@wisc.edu 
the Last Planner ${ }^{\circledR}$ System (Ballard 2000) and Location-Based Management System (Kenley et al. 2006). This paper builds on the combination of these two systems (Seppänen et al. 2010) through proposing an integrative production system, consisting of five cohesive streams. This production system is presented in the context of the Van Ness and Geary Campus (VNGC) Hospital project, located in San Francisco, California.

\section{PRoJECT CONTEXT}

The VNGC hospital project is an ongoing megaproject that is being executed through an Integrated Lean Project Delivery ${ }^{\circledR}$ (ILPD) system that is supported by employing an Integrated Form of Agreement (IFOA) and profit sharing. The fundamentals of the employed project delivery system are further explained by Lichtig (2005). The project scope is building a LEED Silver, fifteen-floor hospital that will create 779,508 square feet of diagnostic, treatment, and inpatient bed space, as well as 210,290 square feet of parking space. Through this project, Sutter Health (the owner of the project) will provide 274 licensed patient beds and 435 parking spaces. The total budgeted cost of the project is $\$ 2.03$ billion, with a duration of 68 months, ending in the first quarter of 2019.

The superior performance of the proposed production system is illustrated through the notable performance of the VNGC Hospital project. At the time of writing this paper, the project has been on schedule with a cost performance index (earned labor hours per actual ones) of 1.25. Also, the project team has successfully increased its contingency budget, through savings, by $28.6 \%$ so far. Moreover, the project has been achieving remarkable safety performance; for example, its average worker compensation cost per labor hour has been $\$ 0.06$ per hour, as compared to an industry average of about $\$ 1$ per hour. Following is a holistic overview of the project's production system that has enabled such performance, as captured by numerous project-site visits and extensive interviews of the project team.

\section{Project Production System}

An illustration of the production system, as applied in the VNGC Hospital Project, is depicted in Figure 1, showing its five streams in each of its five implementation stages.

The proposed production system is designed with the main objective of ensuring predictable and streamlined workflow for complex projects. To do so, the production system is structured into five cohesive streams, each of which is divided into five different implementation levels. This design is intended to promote whole-system thinking, emphasizing that all resources are tied to the same physical output. This is particularly important in the case of megaprojects, in which project participants usually tend to optimize components in isolation, thus hindering the whole project, if these components are not designed to cohesively work with one another. Following is a further explanation of each of the five streams, starting with the production planning stream.

\subsection{Production Planning}

The proposed production planning incorporates the Last Planner ${ }^{\circledR}$ system's stages, with the addition of the "production strategy" stage that corresponds to the "review and optimize" implementation level. Further explanations of the five implementation levels of production planning are presented in the succeeding paragraphs. 


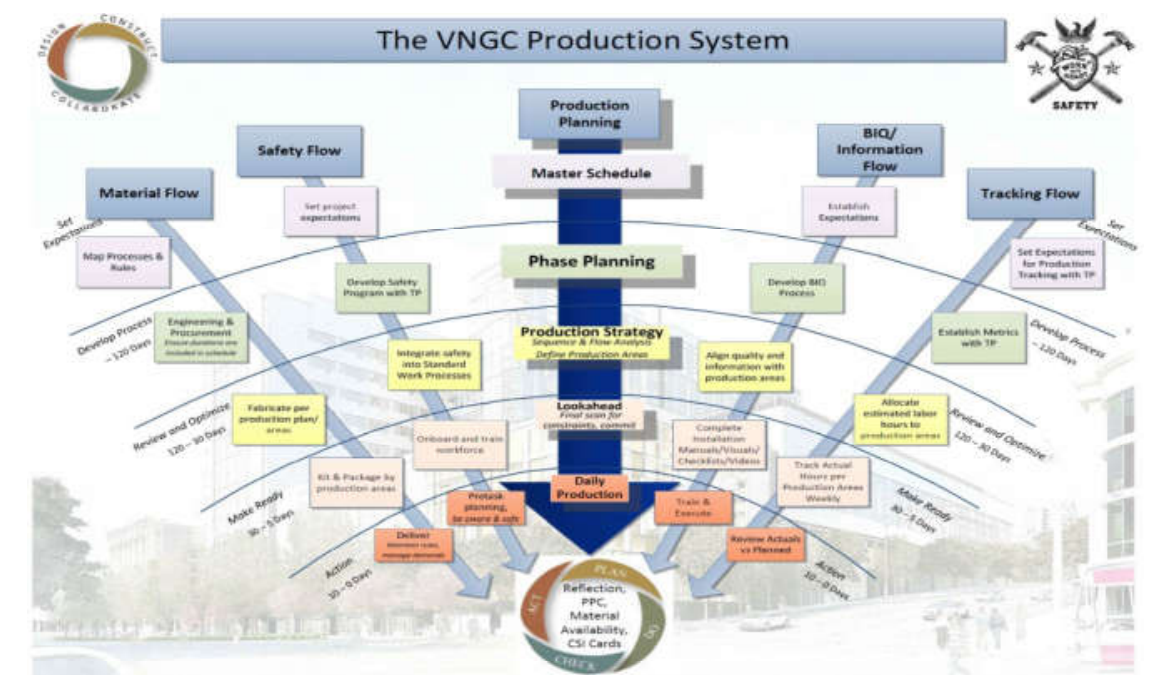

Figure 1: The proposed production system as applied in the VNGC Hospital Project

\subsubsection{Master Schedule}

In the first implementation level, entitled "set expectations," the production planning process is implemented by developing a master schedule incorporating the main project milestones. At this level, the main goal is identifying the goals that satisfy the client's expectations, and reflecting these goals in the project's master schedule. It is critical at this stage to integrate all the relevant estimator's information, since project leaders need such information to comprehend how project drivers and constraints affect planning. Therefore, during this stage, the project team should consider the provided project documents, performance goals, conditions affecting methods and sequence, interface with existing occupancy, special equipment and resources, initial approvals, and long-lead items. The project team should use its relevant experience and refer to comparable project phasing and durations as a guide while reflecting the project goals in the project's master schedule.

\subsubsection{Phase Planning}

In the second implementation level entitled "develop process," the production planning process is implemented by developing phase planning. The goal of this stage is to establish the project phases and their milestones, while making them visible through handoffs. This goal is achieved by performing five steps. First, the project team coordinates to establish major phases of work while focusing on design and construction phases, agency reviews, long lead items, and system turnovers. During this step, the project team works together in making the relationships of required work between trade partners visible. Second, the project team cooperates in identifying milestones for each phase as well as the conditions of satisfying them. Third, the project team works together in recognizing the work streams (activities by discipline or system) within the developed phases. Fourth, the project team collaborates in highlighting the expected outcomes for segments of works. Fifth, the project team checks and verifies the practicality of the phase planning efforts. Throughout phase planning, it is critical to implement a pull technique, actively engage key trades, and make sure that project superintendents validate the phase plan.

\subsubsection{Production Strategy}

In the third implementation level entitled "review and optimize," the production planning process is implemented by developing a production strategy. The main goals of this stage 
are implementing sequence and flow analyses, defining production areas, and designing production using takt-time principles to achieve stable and predictable construction workflow. These goals are achieved by performing four steps. First, for each phase, the project team investigates different production strategy alternatives, and concludes by choosing a preferred strategy. Second, in accordance to the selected production strategy, the project team works on defining general work sequence (in terms of different disciplines). Third, the project team works on clearly defining production areas, so that these areas have almost similar scope. Fourth, the project team implements takt-time planning so that all trades can move through the project at the beat of the same drum. In the VNGC hospital project, at the time of writing this paper, there were 36 work-teams moving through 110 defined production areas at nearly the same rhythm.

\subsubsection{Lookahead}

In the fourth implementation level entitled "make ready," the production planning process is implemented through performing lookahead. To ensure that the planned work is ready to be executed, all constraints are reported and the team works together on solving them. To do so, different team members commit to being the champions of solving specified constraints, and the status of each constraint is regularly reported to the whole project team. For successful application, the project team must lookahead far enough to prevent production interruption, while making the whole process visual and collaborative.

\subsubsection{Daily Production}

In the fifth implementation level entitled "action," the production planning process is implemented through planning and controlling daily production. This stage incorporates two steps. The first step is daily huddling to quickly and effectively adjust for variances. This step is critical as it fosters a collaborative culture onsite while effectively ensuring that all the tasks for that day are constraint free, and that each task is a reliable commitment. The second step is swarming to solve any unforeseen constraint. This step is vital to keeping the plan reliable. At the end of each day, the team records the completion of daily activities, thus enabling the calculation of percent-plan-complete (PPC) to verify the reliability of the plan. In the case of incompletion, the area superintendents and the related trade partners' general foremen apply a five-why-process to pinpoint the root causes, and implement actions to prevent future incompletions.

\subsection{Material Flow}

To achieve predictable and streamlined workflow, the entire supply chain must be aligned with the production flow on site. In megaprojects, this task is extra challenging. The material flow stream of the proposed production plan is designed to resolve this challenge.

\subsubsection{Setting Expectations and Rules}

In this implementation level, the project team works on aligning the material flow to the production plan through linking deliveries to important milestones. Also, during this stage, the project team starts selecting vendors that align with the set expectations, and involving them in the process. Together, the team works on exploring how to optimize the use of pre-fabrication, as well as establishing a visible supply chain monitoring system that can easily reveal variances to plan. It is critical, at this stage, that the team does not jump into detailed planning until expectations are defined and aligned. 


\subsubsection{Engineering and Procurement}

In the second implementation level, the project team focuses on sequencing and prioritizing engineering efforts in the order of fabrication and assembly. The main objective of this stage is empowering engineering to release complete and accurate information to fabrication, as well as optimizing prefabrication and component utilization.

\subsubsection{Fabrication per Production Areas}

In this stage, the project team aligns the fabrication sequence with the order of field installation/demand. The time buffer between fabrication and installation is sized to both minimize storage and reduce material shortage. Also, in this stage, the project team aims at achieving fabrication in a controlled environment to the largest extent possible.

\subsubsection{Kitting and Packaging by Production Areas}

In this stage, all materials are kitted and clearly labelled to match production areas. To complement the kitting and packaging of materials, any specialized tools, as needed, should be included. It is important to kit materials and deliver them on wheels to minimize dunnage and transportation waste.

\subsubsection{Delivering, Maintaining Rules, and Managing Demands}

In this stage, materials are delivered in the order of installation. All deliveries should be coordinated and recorded, and any unscheduled deliveries should be sent away if they have the potential of disrupting any planned deliveries. During this stage the team should work on eliminating waiting time and double handling.

\section{$3.3 \mathrm{BIQ} /$ Information Flow}

Another important aspect of achieving and maintaining predictable and streamlined workflow is having the right information available for the right people at the right time to enable building-in-quality from the first time. Following is a further explanation of the five implementation levels of BIQ/information flow.

\subsubsection{Establishing Expectations}

In this stage, the project team identifies the customers' quality expectations, and converts them to requirements. Also, the team identifies the related trade partners' superintendents, Quality Assurance/Quality Control (QA/QC) manager(s), and Project Managers (PMs), as well as special inspector(s), if applicable, and empowers them throughout the process.

\subsubsection{Developing BIQ Process}

After identifying the quality expectations as well as the key people, the team collaborates in developing a standard process that consistently produces work based on the identified customers' expectations and the countermeasures that can prevent past failures, as well as the approved drawings, specifications, and Testing, Inspection and Observation (TIO) requirements (if applicable). The objective of this step is to clearly map the trade process after capturing all the steps, lessons learned, and best practices to complete each step safely and successfully. During this stage, it is critical to interact and establish relationships with trade field-leads.

\subsubsection{Aligning Quality and Information with Production Areas}

During this stage, the construction team is provided with approved documents and field checklists that helps assure conformance to expectations, as per each production area. Then, the construction team reviews the scope, as well as the related process map and the 
quality considerations identified for each step, and validates that the Inspection Request (IR) batches align with installation. Also, during this stage, the team works on eliminating any duplication while still meeting inspection requirements.

\subsubsection{Completing Installation Manuals/Visuals/Checklists/Videos}

During this stage, the construction team tests the developed BIQ plan, pre-inspection checklists, and implement improvements. If applicable, the team, additionally, finishes and distributes First Run Study (FRS) documentations. The objective of this stage is finalizing the confirmation that the deigned process aligns with the approved information.

\subsubsection{Training and Executing}

During this stage, the production team ensures that all field-workers have all necessary information developed in the implemented BIQ program, and are using the pre-inspection checklists as intended. During this stage, active interaction (e.g. weekly check-ins) with trade field-leads is essential to capture any variances to plan, and to make sure that appropriate learnings are shared and adjustments are made instantly.

\subsection{Tracking Flow}

Tracking performance is essential for achieving project success. Since the construction industry is labor-intensive, labor performance tracking is vital to ensure a smooth workflow, as well as to identify and reduce risks. In megaprojects, this task is extra challenging. The tracking flow stream of the proposed production plan is designed to resolve this challenge as illustrated in the following paragraphs.

\subsubsection{Setting Expectations for Production Tracking with Trade Partners}

In this stage, the project team defines expectations and set goals regarding production tracking. It is essential to engage all trade partners in this collaboration to clearly define expectations and incorporated assumptions, and to attain commitment from key members. For example, in the VNGC hospital project, this stage concluded that the team will track the project the way it will be built. In this sense, the team has committed that with the completion of each production area, the team will follow a defined assessment process to evaluate how this production area was completed, and asses the overall performance trend.

\subsubsection{Establishing Metrics with Trade Partners}

After setting the expectations for production tracking, the production team collaborates with each trade partner to establish clearly defined metrics. Overall, these metrics should be sufficient to evaluate the performance of on-site field labor, off-site fabrication labor, non-direct labor, trade specific productivity, and material tracking.

\subsubsection{Allocating Estimated Labor Hours to Production Areas}

In this stage, field leadership of the trade partners collaborate with the production team in allocating the estimated labor hours to the defined production areas. Together, they report these numbers using visual area maps.

\subsubsection{Tracking Actual Hours per Production Area}

In this stage, the field leadership of the trade partners collaborate with the production team in tracking the actual labor hours for each active production area.

\subsubsection{Reviewing Actuals vs Planned}

Having both the estimated labor hours as well as the actual ones for each discipline within the active production areas, the project superintendents and PMs collaborate with the 
production team in assessing the achieved performance by comparing the actuals vs the planned. If this process reveals any insufficient performance, together the area superintendents and the trade partners' general foremen apply a five-why-process to pinpoint the root cause(s), and implement actions to prevent future failures. On the other hand, the main factors that have enabled remarkable performance are recorded as lessons learned to be shared throughout the project.

\subsection{Safety Flow}

In the proposed integrative project production system, safety is an equally vital aspect and is carefully incorporated in every step of production. Unfortunately, megaprojects often experience numerous safety incidents. Following is an explanation of the five implementation levels of the safety flow stream that is designed to face this challenge.

\subsubsection{Setting Project Expectations}

In this stage, the project team collaborates to form a dedicated safety core group. This safety core group collaborates with the project directors and trade partners' PMs to define measurable safety goals (such as lost time incident rate, total incident rate, and recordable rate, among others), as well as the targeted safety-related behavior and overall culture.

\subsubsection{Developing Safety Program with Trade Partners}

During this implementation level, the safety core group collaborates with the project's superintendents, foremen, and safety managers in four main tasks. First, the development and implementation of safety into the design phase. Second, the development and implementation of loss time prevention policies. Third, the development and implementation of procedures ensuring compliance with applicable rules and regulations of all federal, state, and local agencies. Fourth, the development and implementation of phase-specific safety enhancements.

\subsubsection{Integrating Safety into Standard Work Processes}

During this implementation level, all project superintendents work on integrating safety into standard work processes. In the VNGC hospital project, this is achieved through four avenues. The first is preconstruction meetings, in which superintendents identify safety concerns during production planning. The second is weekly (every Monday) site safety boost assembly, in which superintendents share with all the workers the relevant information about the identified hazardous activities occurring this week, and how to execute them safely. The third is weekly coordination meetings (every Friday) in which superintendents work on identifying safety concerns for the following week. The fourth is daily huddles, in which superintendents review daily safety issues with trade field-leads.

\subsubsection{Onboarding and Training Workforce}

During this implementation level, project superintendents and foremen work together on three main tasks. First, ensuring that all field staff are given basic and site-specific trainings. Second, making sure that all workers are aware of current hazards in the field and know where to go for additional information and help. Third, confirming that all staff have proper Personal Protective Equipment (PPE), and know how to us it. Additionally, it is critical to involve and fully engage new hires in the established safety culture. For example, in the VNGC hospital project, new hires are required to watch a short video about the project in which project workers, managers and executives welcome them while promoting the project culture, specifically safety culture. 


\subsubsection{Pre-task Planning}

In this stage, everyone on the project must be actively using the developed safety-related resources. For example, in the VNGC hospital project, all field workers fill in safety-related information in the Pre-task Handbook to review activity-based safety protocols, and use Continuous Safety Improvement (CSI) Cards to recognize additional safety-related issues.

\section{CONCLUSION AND RECOMMENDATIONS}

This paper proposes an integrative production system for megaprojects. This production system consists of five cohesive streams, each of which has been explained throughout the paper in the context of the ongoing construction work of the VNGC hospital. It is vital to note that many success-pillars must be established for the proposed production system to flourish. The four most important pillars, as referred by the VNGC hospital project team, are: a truly collaborative culture that is based on respect for people and aligned interests, a people-empowering culture that fosters care and innovation, a continuous improvement culture that targets perfection, and colocation that promotes a collaborative problem solving culture. To promote these success pillars, the VNGC hospital project is being executed through an ILPD ${ }^{\circledR}$ system that is supported by employing IFOA and profit sharing. Moreover, to advance the success potential of the proposed production system, the VNGC hospital project has been implementing Building Information Modeling (BIM), not as a sixth stream, but as a tool of the production system that further enhances its performance and cohesiveness. It is important, however, to note that implementing such rigorous production system can result in making the project increasingly intolerant for variances and changes. This is a potential growth area for the proposed production system that should be addressed by future research efforts.

\section{ACKNOWLEDGEMENTS}

The authors would like to thank team members from Sutter Health, The Boldt Company, Herrero Builders, SmithGroupJJR, and the trade partners of the VNGC hospital project for their active participation, and for sharing their processes and best practices.

\section{REFERENCES}

Ballard, H.G., 2000. The last planner system of production control (Doctoral dissertation, The University of Birmingham).

Changali, S., Mohammed, A., and Nieuwland, M.O., 2015. The construction productivity imperative. McKinsey Productivity Sciences Center.

Hanna, A.S., 2016. Benchmark Performance Metrics for Integrated Project Delivery. Journal of Construction Engineering and Management, p.04016040.

Hanna, A.S., Ibrahim, M.W., Lotfallah, W., Iskandar, K.A. and Russell, J.S., 2016. Modeling Project Manager Competency: An Integrated Mathematical Approach. Journal of Construction Engineering and Management, p.04016029.

Ibrahim, M.W., 2016. Modeling, Benchmarking, and Maximizing Project Delivery Performance (Master's Thesis, University of Wisconsin-Madison).

Kenley, R. and Seppänen, O., 2006. Location-based management for construction: Planning, scheduling and control. Routledge.

Lichtig, W.A., 2005. Sutter health: Developing a contracting model to support lean project delivery. Lean Construction Journal, 2(1), pp.105-112.

Seppänen, O., Ballard, G. and Pesonen, S., 2010. The combination of last planner system and location-based management system. Lean Construction Journal, 6(1), pp.43-54. 